\title{
Die Gerusalemme liberata-Serien von Antonio Tempesta im Kontext des Kulturaustausches zwischen Rom und dem ,Orient' um 1600
}

Die Untersuchung ,kultureller Rahmen' ist in der Kunstgeschichte dann sinnvoll, wenn sie dazu dient, gleichsam aus dem Studium des Ornaments der Rahmenleisten zusätzliche Informationen für ein besseres Verständnis des vom Rahmen eingefassten Kunstwerks zu erhalten. Ein solcher Ansatz scheint zur Weiterentwicklung der kunsthistorischen Beschäftigung mit den Radierungen Antonio Tempestas zur Gerusalemme liberata Tassos tatsächlich angebracht.

Die Chronologie und die elementaren Umstände von Produktion und Publikation der drei Gerusalemme-Zyklen Tempestas dürfen als geklärt gelten ${ }^{1}$ : Sie sind zwischen ca. 1605/06 und den späten 1620er Jahren entstanden, datieren also deutlich später als die Tasso-Kupferstiche von Agostino Carracci nach Bernardo Castello. Anders als bei Castello sind direkte Kontakte Tempestas mit Tasso nicht belegt. Dennoch ist bekannt, dass sich Tempesta mindestens gelegentlich im Ambiente der römischen Förderer und Bewunderer des späten Tasso bewegt hat, denn er arbeitete für Cinzio und Pietro Aldobrandini, die Nepoten von Papst Clemens VIII., denen er jeweils auch druckgraphische Serien dedizierte. ${ }^{2}$ Obwohl also Tempestas Verbildlichung der Dichtungen Tassos kaum direkt auf einen intensiven Austausch mit diesem oder konkrete Anweisungen des Poeten zurückgehen dürfte, bieten die Graphiken und ihre Legenden Aufschluss sowohl zum Verhältnis von Text und Bild in der Zeit Tempestas (wozu Italianisten selbstverständlich mehr sagen können) als auch ganz besonders zu einigen allgemeineren kulturellen Voraussetzungen dieser Darstellungen.

Antonio Tempestas Karriere als Illustrator von Tassos Dichtwerken begann 1607, als der römische Verleger Giovanni Angelo Ruffinelli eine Gerusalemme herausbrachte, die zwanzig kleine Radierungen enthielt, eine vor jedem Gesang. ${ }^{3}$ Von dieser handlichen Ausgabe gab es unveränderte Wiederauflagen in den Jahren 1621, 1627 und noch viel später, als die Platten bei der Verlegerdynastie De Rossi gelandet waren. Tempestas Drucke für Ruffinelli folgen mehr oder weniger direkt einer Serie von Holzschnitten in der 1604 in Genua publizierten Gerusalemme liberata des Verlegers Giuseppe Pavoni, die ein unbekannter Holzschneider nach Zeichnungen von Bernardo Castello angefertigt hatte. Selbst wenn Tempesta Skrupel angesichts eines solchen Plagiats gehabt haben sollte, hätte sein Verleger Ruffinelli ihn entschuldigt, denn die Genueser Ausgabe war, 
was den Text angeht, offenbar eine unautorisierte Edition seiner eigenen - unillustrierten - Gerusalemme, die er 1601 in Rom publiziert hatte. ${ }^{4}$ Die offenbar hohe Nachfrage nach illustrierten Gerusalemme liberata-Editionen scheint Ruffinelli dazu bewogen zu haben, durch Indienstnahme von Tempesta seinen Anteil am Markt zu erobern. Vorzeichnungen wurden bislang nicht gefunden, waren für so kleine und konzentrierte Kompositionen vielleicht auch nicht nötig. Giambattista Marino, passionierter Sammler der Radierungen Tempestas, schrieb Lorenzo Scoto 1622: „Mi manca solo la picciola Gierusalemme del Tasso istoriata da lui, né so dove trovarla".5 Marino muss hiermit Tempestas erste Gerusalemme Serie gemeint haben, die offenbar selten als Sammlergraphik außerhalb von Textausgaben zirkulierte.

Die beiden späteren Tasso-Zyklen Tempestas aus den 1620er Jahren sind - nach den Metamorphosen, den Alttestamentlichen Schlachten und den Batavern - typisches Produkt der Orientierung seines Alterswerks auf literarische Themen. Beide waren nicht an eine Buchausgabe des Gedichts gebundenen und schon daher größerformatig als die Ruffinelli-Radierungen. Die frühere der beiden Serien ist undatiert, trägt am Anfang aber eine Widmung des Künstlers an Taddeo Barberini (1603-1647), die mit dessen Familienwappen und zwei Personifikationen verziert ist. Dieser insgesamt ,kunsttheoretischer $^{`}$ als frühere Dedikationen Tempestas angelegte Text, den Nicolas Poussin für eine seiner Widmungen ausplünderte ${ }^{6}$, kann wegen der für sein Spätwerk typischen gelehrten Aspirationen (und wegen der Vermeidung des Lateinischen) als vom Künstler selbst verfasst gelten. Die Widmung an Taddeo ist ein wichtiges Belegstück für die schon von Giulio Mancini und Giovanni Baglione gerühmte sprachliche Eleganz und geschliffenen Umgangsformen unseres „Florentinus“. Tempesta reflektiert in seinen Worten den zeitgenössischen Diskurs zum Verhältnis von Literatur und bildender Kunst, mit dem er durch die vorliegende Umsetzung eines volkssprachlichen Dichtwerks in Radierungen zwangsweise konfrontiert war. Seine Gedanken zur Spannung zwischen der Wirklichkeit und ihrer literarischen oder bildlichen Darstellung fasste er in die Form des Herrscherlobs: Im Gegensatz zur papiernen Existenz der vom Künstler gezeichneten Figuren („figure insensibili de azzioni buone e virtuose“) sei Taddeo eine „figura viva di bonta, e di virtù" und zudem ein Mensch, der sich am disegno erfreue. Indem Tempesta seine radierten Szenen aus Tassos Heldengedicht dann als „quasi un suo ritratto", also als ein indirektes Porträt des Taddeo Barberini, vorstellte, parallelisierte er dessen Übernahme des Patroziniums über die vorliegende Serie als Würdigung des künstlerischen Virtuoso Tempesta durch den moralisch-staatsmännisch definierten Virtuoso Taddeo. Mit diesen Bilddrucken, Ausweisen seiner stummen Kunst, hoffe er, Tempesta, somit, nicht hinter den wortreichen Enkomien auf Don Taddeo zurückzustehen, wie ja zuweilen auch Gott die von Laien in den Kirchen aufgehängten Votivbilder mehr berücksichtige als die von Priestern zu ihm gesprochenen langen Gebete.

Tempestas zweite Gerusalemme liberata-Serie (Abb. 1a und 1b) ist, wie erwähnt, nicht zusammen mit dem Text von Tassos Epos publiziert worden. Anders als die Stiche von 


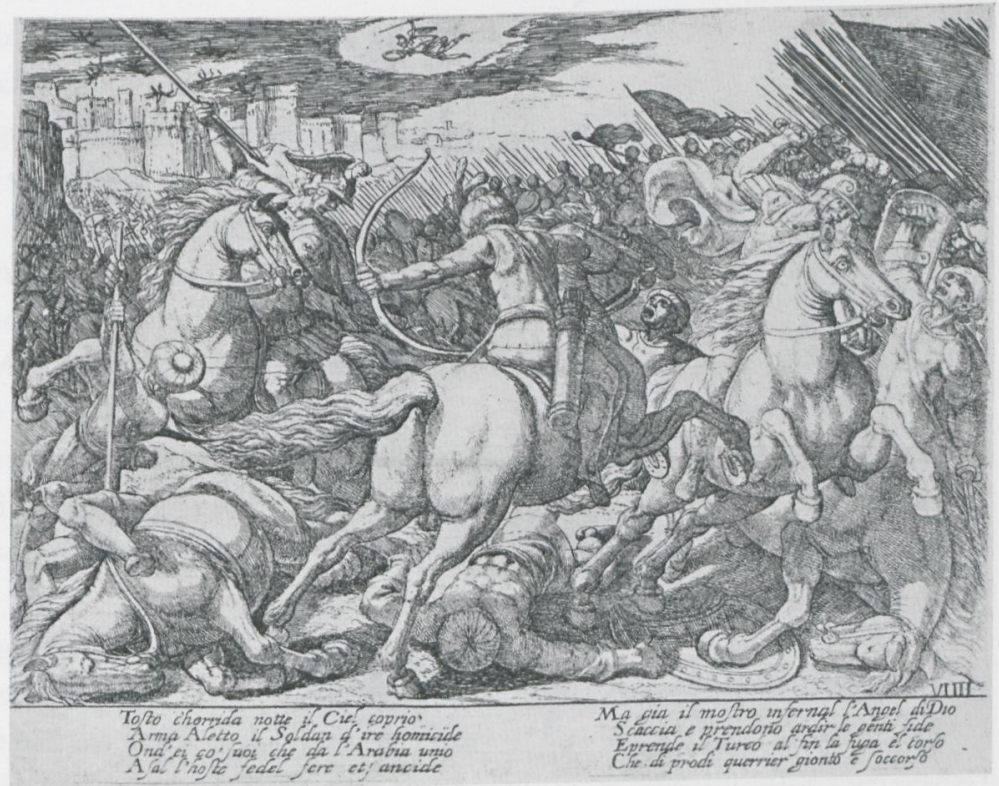

Abb. 1a Antonio Tempesta, Blatt aus der zweiten, Gerusalemmé-Folge,

Privatbesitz, 1620er Jahre.

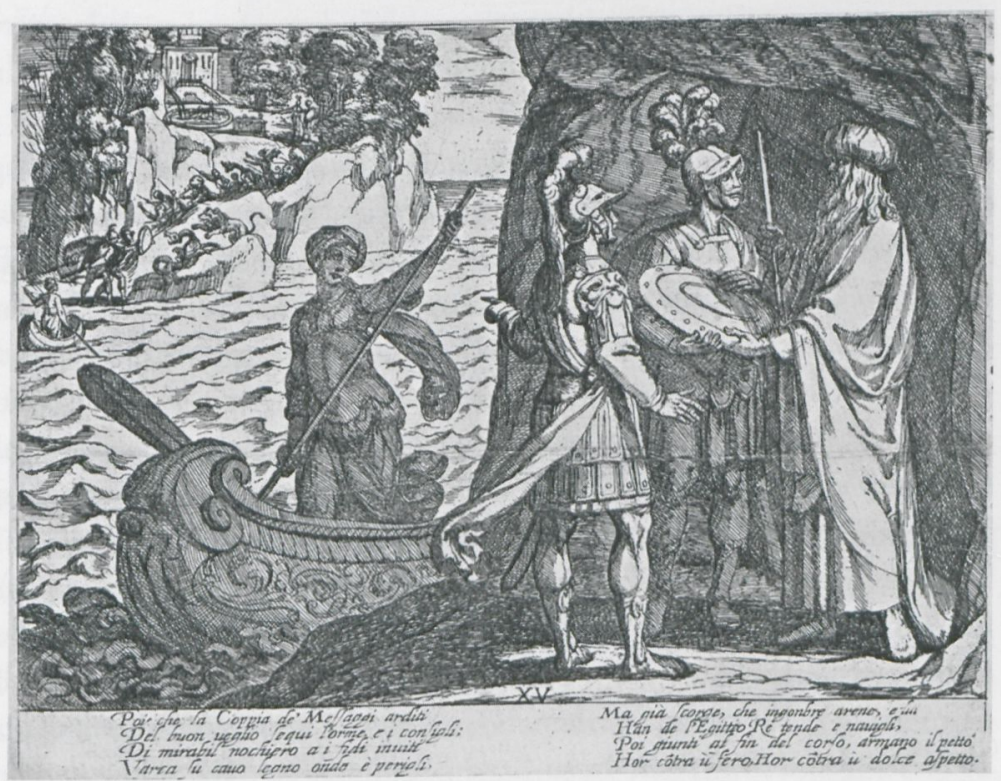

Abb. 1b Antonio Tempesta, Blatt aus der zweiten, Gerusalemme-Folge,

Privatbesitz, 1620er Jahre. 
Agostino Carracci und Giacomo Franco nach Bernardo Castello in der Gerusalemme liberata von 1590, anders auch als diejenigen des Camillo Cungi nach Castello von 1617, wurden seine Radierungen zwar im unteren Rand mit kurzen Legenden versehen, fungierten aber nicht mehr als eine Art zusätzliches visuelles argomento (= kurze Zusammenfassung) des Stoffes, das jedem der zwanzig Bücher Tassos in den klassischen Textausgaben voransteht. Tempestas zweite und dritte Gerusalemme liberata-Serie sind von der ausschließlichen Dienstbarkeit für den Text befreit und damit wesentlich für das Entstehen einer unabhängigen Bildtradition von Tassso-Sujets in Malerei und Graphik. Dennoch blieb die Literatur eine wesentliche Bezugsgröße der Gerusalemme-Serien Tempestas. Nicht nur die gedichteten Unterschriften der einzelnen Darstellungen, sondern auch die von Tempesta selbst in seiner Widmung an Taddeo Barberini bekundete Intention, jeden erfreuen zu wollen, der tutte edue le professioni (= bildende Kunst und Dichtung) in sieme genieße, weisen auf diesen Nexus.

Die zweite Gerusalemme-Serie Tempestas zeigt motivische Abhängigkeiten von Cungis Kupferstichen nach Castello und teilt deren parataktischen Ansatz in der visuellen Umsetzung der Schlüsselepisoden eines jeden Gesangs: Zwar wechselte Tempesta vom Hoch- zum Querformat, doch hielt er an dem vom Genuesen gewählten Prinzip der Simultandarstellung fest. Anders als Castello hat er jedoch in der Mehrzahl seiner TassoRadierungen die Aufmerksamkeit im Vordergrund konzentriert, wo der Betrachter eine kleine Zahl sorgfältig arrangierter Figuren findet. Andere Handlungsebenen sind zuweilen hinzugefügt, aber in weitaus kleinerem Maßstab gezeigt und mit der Hauptszene durch Zeigegesten oder Blickkontakte verbunden. Diese ausbalancierten Kompositionen dürfte Tempesta durch Zeichnungen vorbereitet haben, jedoch sind solche für die Zweite Gerusalemme liberata-Serie bisher nicht gefunden worden.

Eine Verlegeradresse auf dem zweiten Druckzustand des Widmungsblattes an Taddeo Barberini und die Seltenheit erster Zustände beweisen, dass die Platten der zweiten Tasso-Serie offenbar schon kurz nach der ersten Drucklegung in den Besitz des französischen Verlegers François Langlois genannt Ciartres (1588-1647) gelangten, der in den 1620er Jahren mehrfach in Rom war. Der genaue Zeitpunkt des Kaufs der GerusalemmePlatten durch Ciartres ist nicht zu ermitteln, doch kann man das Datum wohl auf die Mitte der 1620er Jahre ansetzen, weil Tempesta nochmals eine ähnliche Tasso-Serie gravierte - eine Prozedur, die nur unter dem Aspekt sinnvoll war, dass sie die ins Ausland gewanderten Platten der Vorgängerserie ersetzte und damit dem Künstler weiterhin Anteil am Verkauf dieser beliebten Sujets ermöglichte.

Für die dritte Gerusalemme Tempestas, die vielleicht zu Lebzeiten des Künstlers nicht mehr erschien, haben sich zwanzig Vorzeichnungen in der Staatlichen Graphischen Sammlung in München erhalten. ${ }^{7}$ Der Künstler scheint sie als seitenrichtige Kopien der Radierungen der zweiten Tasso-Serie begonnen zu haben, hat aber im Laufe des Zeichenprozesses zahlreiche Elemente variiert oder Kompositionen im Sinne der von ihm angestrebten größeren narrativen Stringenz weiter durchgeklärt. Tempestas dritte Tasso- 
Abb. 2 Antonio Tempesta, Blatt aus der dritten ,Gerusalemme-Folge, Privatbesitz, 1620er Jahre.

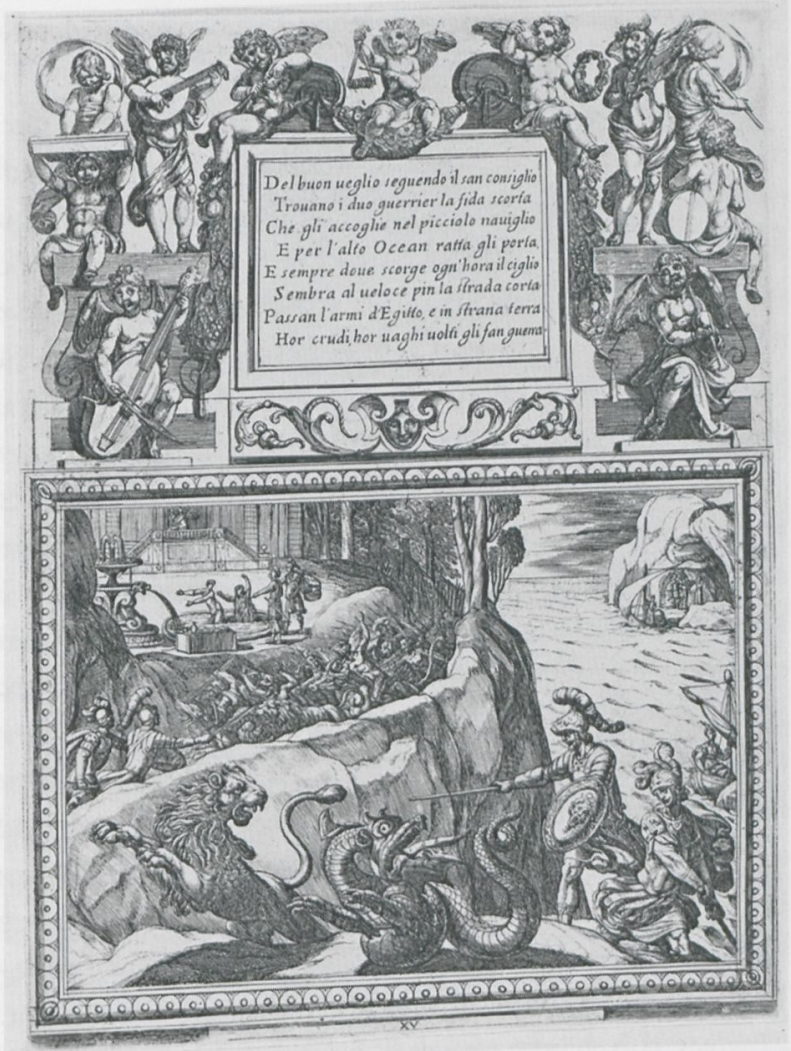

Serie ist somit zwar vor allem eine überarbeitete Version der an Ciartres verkauften zweiten Folge; die einzelnen radierten Szenen wurden aber vom Künstler in große Ornamentrahmen gefügt, die er jeweils über die einzelnen Darstellungen setzte (Abb. 2, Taf. XIII). Anders als in der zweiten Serie entsteht so in der dritten Gerusalemme der Eindruck von radierten Historiengemälden in einer galerieartigen Präsentation, die entfernt an die Stuckrahmen der Galérie François I $^{\mathrm{er}}$ in Fontainebleau denken lässt.

Anhaltspunkt für die Richtigkeit der These einer nicht mehr durch Tempesta selbst erfolgten Edition dieser Serie ist die Notiz des Verlegers Mainardi, der die Drucke im Rahmen einer Textausgabe 1735 in Urbino herausbrachte, dass die Drucke bislang nicht in altra Edizione publiziert worden seien. Dies stimmt zwar so sicher nicht, weil in der Mainardi-Ausgabe das Bild des Canto I schon die Adresse des in Rom tätigen Verlegers Arnold van Westerhout (1651-1725) trägt, doch ist das weitgehende Fehlen von Monogrammen bzw. Signaturen Tempestas oder eines Verlegernamens aus den 1620 er oder 30 er Jahren auffällig. Außerdem gibt es separate Abzüge der für die Legenden benutzten Groteskenornamente, in denen noch kein Text steht. Daher ist denkbar, dass die zwar phantasievollen, aber in den 1620 er Jahren fast schon veralteten Grotes- 
kenformen aus einem vorgängigen Druckprojekt des Künstlers stammen oder zumindest auf weitaus älteren Studien beruhen.

Abgesehen von diesen Details ist im Vergleich der Kompositionen Castellos mit denjenigen der zweiten und dritten Serie Tempestas auffällig, dass letztere weniger statisch und schematisch sind und in ihnen auch die Frequenz der Turbane, Pluderhosen, Krummschwerter, geschweifter Pfeil- oder Bogen-Köcher und anderer ,fremdländischer ' Elemente deutlich höher ist. Dieses Phänomen verlangt eine Erklärung, die sich nicht nur auf die Antonio Tempesta individuell zur Verfügung stehenden künstlerischen Mittel, sondern auch auf die kulturelle Situation im Italien der Zeit um 1600, speziell die zeitgenössische Wahrnehmung des Orients, beziehen muss.

Es ist bemerkenswert, dass in der Kunstgeschichte bisher wenig erörtert wurde, auf welche Weise in den von Tassos Dichtungen inspirierten bildlichen Darstellungen die christlichen Kämpfer des ersten Kreuzzugs von ihren als Glaubensfeinde, Heiden, Ungläubige etc. etikettierten Gegnern abgegrenzt werden. ${ }^{8}$ Dieses Manko mag einerseits damit zu erklären sein, dass die Dichtungen Tassos noch immer vorrangig als Vertreter einer Gattung gelten, die ureigenen europäisch-,abendländischen ' (Literatur-) Traditionen angehört, etwa dem lateinisch-griechischen Epos oder dem Ritter- und Heldengedicht aus Mittelalter und Renaissance, so dass folglich angenommen wird, auch Bilder mit Gerusalemme-Sujets seien eher aus Versatzstücken vorgängiger Visualisierungen von Troja-, Aeneas- oder RolandsDichtungen zusammengesetzt. Beides ist keineswegs falsch, denn wie Tasso selbst reichlich aus Homer, Vergil und Ariost schöpfte, so scheinen viele Gerusalemme-Bilder des 17. und 18. Jahrhunderts ohne allzu viel Bemühen um Originalität Versatzstücke von Darstellungen gängiger, d.h. häufig gemalter, Sujets aus Mythos und Historie zu verwenden.

Während aber von Seiten der Italianistik ein erster bedeutender Versuch vorliegt, Tassos Gerusalemme-Dichtungen auch hinsichtlich der ,Heidendarstellung' und deren zeitgenössischer Motivationen zu untersuchen, nämlich das Buch von Teresa Staudacher?, finden sich in der kunstgeschichtlichen Forschungsliteratur, wenn es um die kulturellen Kontexte für Gemälde oder Graphiken mit einschlägigen Motiven geht, meist nur allgemein gehaltene Hinweise auf die Türkenkriege. Dabei muss deutlich sein, dass die Dichtungen Tassos in den Jahren der Kämpfe des christlichen Europa mit den Osmanen, speziell kurz vor und nach der Schlacht von Lepanto, entstanden sind, inmitten eines Konflikts also, der in der zweiten Hälfte des 16., im gesamten 17. und teils noch im frühen 18. Jahrhundert die politische Bühne Europas beherrschte und der fraglos auch bei der Betrachtung künstlerischer Darstellungen literarisch fixierter Ereignisse aus der historisch fernen Kreuzfahrerzeit mitassoziiert wurde. Dies, obwohl die Machtverhältnisse zu Lebzeiten Gottfrieds von Bouillon noch ganz andere waren als im späten Cinquecento, also das Heilige Land nicht unter Kontrolle der Türken stand; denn ähnlich wie bei Tasso weitete sich der aktuelle militärische Konflikt in vielen Interpretationen des späteren 16. und frühen 17. Jahrhunderts zu einem grundsätzlichen Antagonismus von Christen- und Heidentum. ${ }^{10}$ 
Abb. 3 Antonio Tempesta, Modellzeichnung für das Relief zu Ehren von

Marcantonio Colonna als Sieger der Seeschlacht von Lepanto, Paris, Musée du Louvre, ca. 1590.

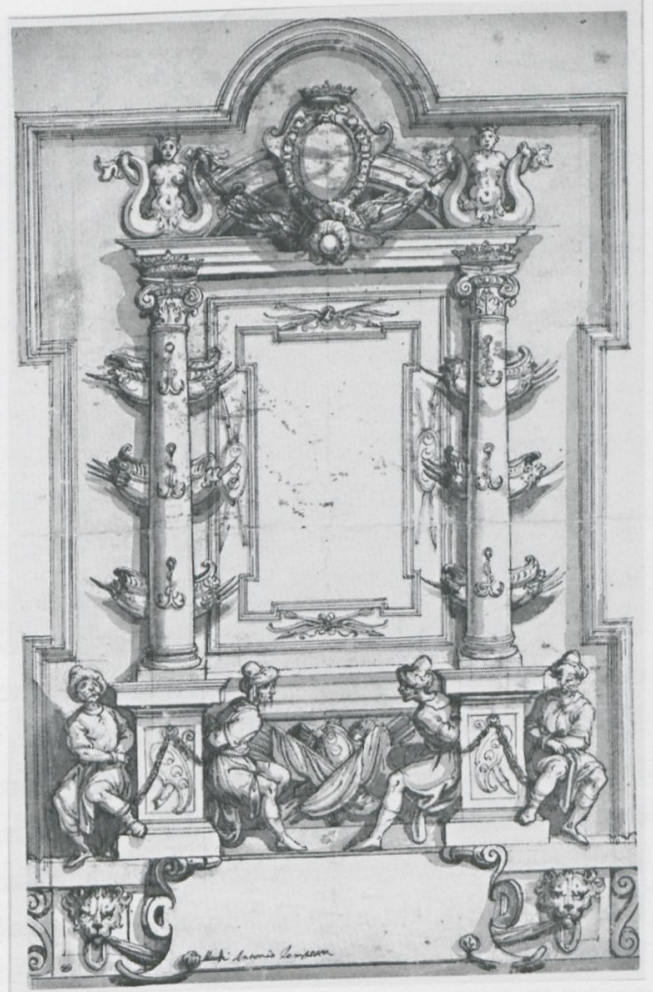

Es ist kein Zufall, dass - neben den Hofmalern Kaiser Rudolfs II. ${ }^{11}$ - wenige Künstler der Epoche die genannten kriegerischen Auseinandersetzungen mit den Osmanen so intensiv wie Antonio Tempesta dargestellt und im Sinne eines fundamentalen kulturellen und/oder religiösen Gegensatzes ausgedeutet haben. Tempesta tat dies einschließlich der Stereotypen und Widersprüche einer zwischen Furcht, Hass und Bewunderung (meraviglia ${ }^{12}$ ) schwankenden Stimmungslage im christlichen Europa. Sein Engagement erklärt sich wesentlich aus seiner Dienstbarkeit für wichtige Instanzen der römischen Kirche und des Adels. Mehrfach griff Tempesta dabei Bildformeln auf, die er in triumphalistischen Dekorationen finden konnte, die seine künstlerischen Vorgänger und Anreger im Vatikan und andernorts ausgeführt hatten, etwa Giorgio Vasaris Allegorie auf die Seeschlacht von Lepanto in der Sala Regia, die den Sieg der christlichen Liga gegen die Osmanen 1571 durch Hinzufügung einer allegorischen ,B-Ebene` als Überwältigung höllischer Dämonen durch die Kräfte des christlichen Glaubens feiert. ${ }^{13}$ Auch den Topos des besiegt und gefesselt am Boden hockenden osmanischen Soldaten mit Turban, Pluderhosen und markantem Schnurrbart konnte Tempesta bei Vasari finden; er brachte ihn u. a. im Entwurf zum Ehrenrelief für den Sieger von Lepanto, Marcantonio Colonna, im römischen Konservatorenpalast (Abb. 3) an $^{14}$, außerdem in Stechervorlagen für Titel- 
blätter einschlägiger Bücher, z. B. Giacomo Bosios Istoria della Sacra Religione et Ill.ma Militia di S. Giovanni Gierosolimitano von $1602 .{ }^{15}$ Besiegte, Orientalen' schmücken sogar den Helm des antiken Imperators Titus in Tempestas Serie der Ersten Zwölf Römischen Kaiser $^{16}$, was verdeutlicht, dass das Bild des (gefesselten) Turbanträgers auch dazu diente, lange zurückliegende historische Ereignisse zu illustrieren, etwa die Triumphe des römischen Kaiserreiches über ,Barbaren' des Ostens. Auf diese Weise entstand - nicht formal, aber inhaltlich - eine Kontinuität zu antiken Monumenten der römischen Kaiserzeit, in denen der Gegensatz von siegreichen Imperatoren und besiegten Markomannen, Quaden, Jazygen etc. bereits deutlich durch Gewandung oder Haartracht herausgestrichen ist. ${ }^{17}$

Neben solchen direkt auf die römische Antike bezogenen Darstellungen schuf Tempesta auch Bilder mittelalterlicher Konfrontationen christlicher und muslimischer Volksgruppen oder Heere, die für die christliche Seite siegreich waren, vor allem die Schlacht von Las Navas de Tolosa, in der gemäß der Überlieferung schon der Anblick des dem spanischen Trupp vorangetragenen Kreuzes ausreichte, um die Mauren in die Flucht zu schlagen ${ }^{18}$, oder die umfangreiche, im Konflikt der christlichen Spanier mit den Mauren angesiedelte Geschichte der Infanten von Lara (konzipiert zusammen mit dem Rubens-Lehrer Otto van Veen ${ }^{19}$. In diesen Darstellungen von Jahrhunderte $\mathrm{Zu}^{-}$ rückliegenden historischen bzw. halblegendären Begebenheiten werden die Heiden vom Künstler mit Kleidungsstücken und Waffen ausstaffiert, die stark an das oben beschriebene Osmanen-Stereotyp erinnern. In den Schlachten des Alten Testaments, also für die Verbildlichung einer noch weiter als die Römerzeit zurückliegenden Epoche, stattete Tempesta hingegen beide Seiten - die Israeliten und ihre Feinde - mit Turbanen oder anderen ,orientalischen' Kopfbedeckungen (insbesondere konische Mützen mit dekorativ aufgesteckten Federn) aus, auch wenn er die militärischen Führer der Israeliten tendenziell eher wie römische Feldherren gerüstet darstellte. ${ }^{20}$ In seiner Widmung pries Tempesta Großherzog Cosimo II. de' Medici und die Cavalieri di Santo Stefano als Wiedergänger der israelitischen Heerführer des Alten Testaments. Trotz dieser vom Künstler gelegten Spuren in die Toskana und ins antike Rom bleibt unverkennbar, dass die immerhin in Ägypten, Palästina und Syrien angesiedelten Szenen nach Auffassung Tempestas ein Mindestmaß an Lokalkolorit erforderten, das er nicht nur durch Einfügung von Kamelen, sondern auch durch Ausstattung möglichst vieler Beteiligten mit Turbanen etc. herstellen wollte. Auf andere im Rom und Florenz der Zeit verbreitete Elemente der Darstellung osmanischer Krieger verzichtete er allerdings durchweg, sogar in den Gerusalemme-Radierungen: U.a. fehlen bei ihm die kahl rasierten Köpfe und die spitz zulaufenden Sturmhauben. ${ }^{21}$

Dabei darf man vermuten, dass Antonio Tempesta besser als mancher seiner zeitgenössischen italienischen Künstlerkollegen über Kleidung und Gebräuche der muslimisch geprägten Kulturgebiete seiner Zeit informiert war: Gemeint ist seine Arbeit als Entwerfer von Illustrationen und Ornamenten für den in Rom ansässigen, damals europa- 
Abb. 4 Leonardo Parasole nach Antonio Tempesta,

Christus und die Ehebrecherin (im Arabischen Evangelium der Typographia Medicea), Privatbesitz, zweite Hälfte 1580er Jahre.

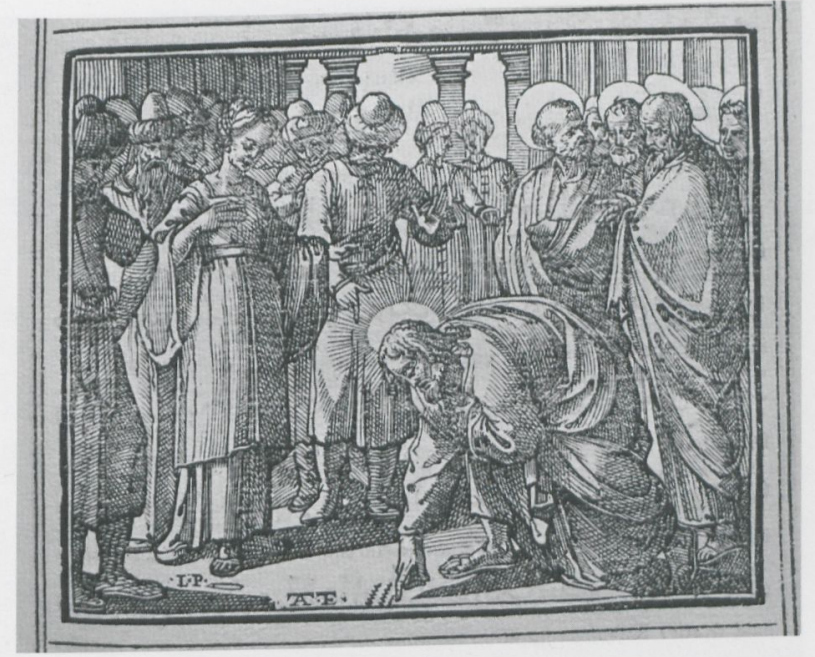

weit führenden Verlag für Drucke auf Arabisch, Syrisch/Chaldäisch und (in Planung) Persisch: die Typographia Medicea Orientale (im Folgenden: TM), gegründet 1584 auf Wunsch von Papst Gregor XIII. und finanziert durch Kardinal Ferdinando de' Medici. ${ }^{22}$ Die TM mit ihrem Direktor Giovanni Battista Raimondi (gest. 1614) sollte einerseits der christlichen Mission dienen, andererseits aber auch Klassiker der arabischen Medizin, Mathematik, Geographie etc. originalsprachlich herausbringen und sowohl im christlichen Europa als auch in arabischsprachigen Regionen mit Gewinn verkaufen. Beide Ansätze setzten intensive Kommunikation mit den ,Zielgebieten 'voraus. Die erstgenannte Absicht, Mission, erschließt sich besonders aus den Arabischen (und Arabisch-Lateinischen) Evangelien der TM. Für die Komposition der von Leonardo Parasole ausgeführten Holzschnitte in diesen Evangelien war Tempesta verantwortlich: Unverkennbar sind hier, etwa in Christus und die Ehebrecherin (Abb. 4), die Gegner Christi bzw. Heiden durch Turbane bezeichnet. Auch für den von der TM verlegten Trattato delle piante et immagini de sacri edifizi di Terra Santa des Franziskanermönchs Bernardino Amico schuf Tempesta Radierungen (1609/10). ${ }^{23}$ Das Buch ist König Philipp III. von Spanien gewidmet, speziell in dessen Eigenschaft als König von Jerusalem. Ausdrücklich wird Philipps aktuelles Engagement im Dienste des christlichen Glaubens hervorgehoben, zumal er sich gerade jetzt durch die Vertreibung der Morisken aus Spanien verdient mache. Wie selbstverständlich enthält der Tafelteil der Terra Santa auch eine Darstellung des Grabes von Gottfried (Abb. 5).

Die TM ist aber keineswegs auf ihre Funktion als flankierende Maßnahme des Anti-Häresie-Kurses bestimmter christlicher Herrscher oder der katholischen Kirche zu reduzieren. Nicht zuletzt das erhaltene Tagebuch Raimondis (in welchem der Direktor übrigens auch den Tod Tassos notierte ${ }^{24}$ ) erweist den Verlag, in dem u. a. konvertierte 


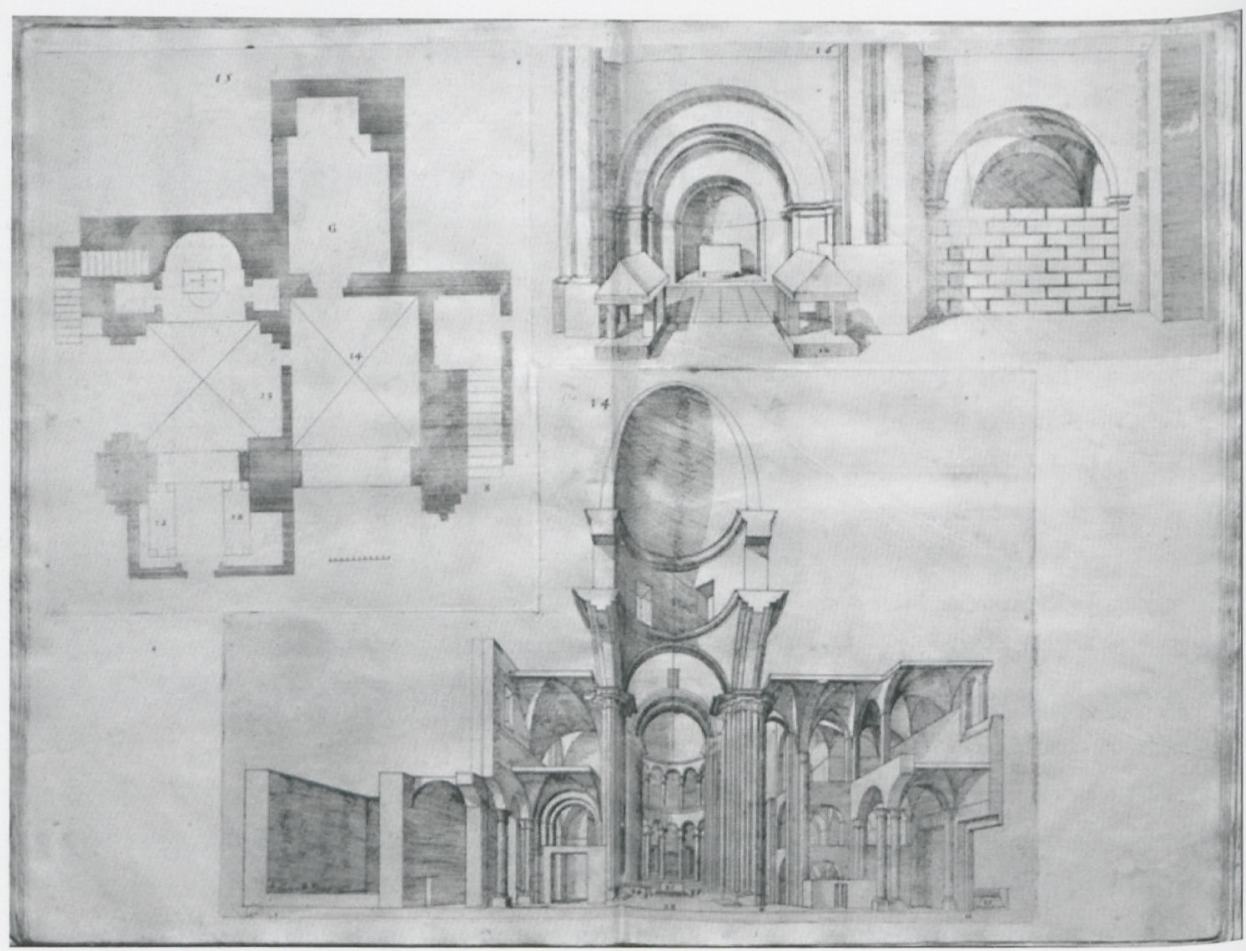

Abb. 5 Antonio Tempesta, Das Grab Gottfrieds von Bouillon in der Grabeskirche in Jerusalem (in der Terra Santa des Bernardino Amico), Rom, Bibliotheca Hertziana, vor 1609.

Muslime arbeiteten, als wichtigen Kommunikationsort Roms mit einem Teil der Welt, der neben den in den Jahren um 1600 bekämpften Osmanen beispielsweise die Perser umfasste, deren Herrscher man sowohl in Rom und Florenz als auch am Habsburgerhof als Verbündete zu gewinnen trachtete; dahinter stand die Idee der Eröffnung einer doppelten Front gegen die Osmanen. Regelmäßig vermerkte Raimondi die Ankunft oder Abreise der Botschafter des Schahs von Persien, Abbas I. (reg. 1587-1629), und anderer außereuropäischer Emissäre in Rom. In den gestochenen und gemalten Bildern dieser in urbe auftretenden Botschafter (Abb. 6), deren Reihe bis zu dem berühmten, von Antoon van Dyck gemalten Porträt des in Diensten der Safaviden stehenden Engländers Robert Shirley (1622, heute Petworth House, Sussex) reichen sollte ${ }^{25}$, erkennt man das sich verstärkende Interesse, gar die Bewunderung für die Kultur derjenigen Territorien, die von solchen mit Turban und kostbaren Gewändern bekleideten Dargestellten vertreten wurden. Nebenbei bemerkt hielt die Angst vor den Osmanen viele Zeitgenossen im christlichen Europa nicht davon ab, die Schlagkraft und hervorragende Organisation der Truppen des Sultans und seines Staatswesens insgesamt zu rühmen und ihren Herrschern als Vorbild zu empfehlen - Motto: von den Türken lernen. Dies gilt unter anderem 
Abb. 6 Unbekannter Stecher

(Orlandi exc.), Der persische Botschafter und seine Delegation vor Papst Paul V., Wien,

Albertina, wohl 1609.

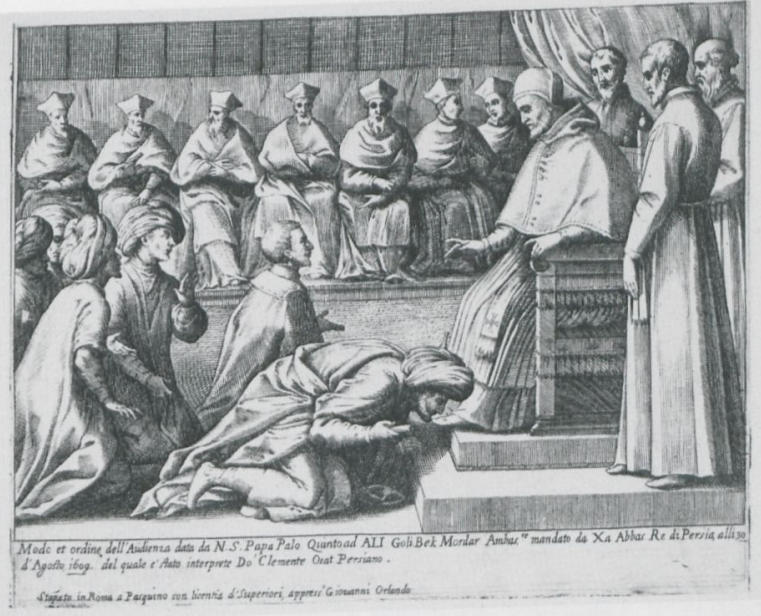

für Scipione Ammirato. ${ }^{26}$ Wenn daneben, wie in den Paralleli militari von Francesco Patrizi, zur Verbesserung der militärischen Schlagkraft gegen die Osmanen das Studium antiker (griechischer oder lateinischer) Strategieliteratur empfohlen wurde ${ }^{27}$, erinnert dies an die charakteristische Parataxe antik-römischer und ,orientalischer' Motive in den oben besprochenen Stichen Tempestas und in den Dichtungen von Tasso selbst. Nicht zuletzt die Mischung zeitgenössisch-,europäischer', ,römisch-antiker' und ,asiatischer Jagddarstellungen in den zahlreichen Jagdzyklen Tempestas deutet darauf, dass der zeitgenössische religiöse und/oder politische Antagonismus zwischen (katholischem) Christentum Europas und den Osmanen in der Zeit um 1600 nicht als unüberwindliche Barriere für Kontakte zwischen beiden Kulturkreisen empfunden wurde. ${ }^{28}$ Solche in der Kunst konservierten Ansätze zu einem Orientalismus noch - weitgehend - ohne Kolonialismus müssen genauer als bisher studiert werden.

Tassos Bearbeitung der überlieferten Ereignisse um den Ersten Kreuzzug ist Dichtung; das Werk konnte und wollte weder streng historische Dokumentation noch Propagandainstrument der Tagespolitik sein. Sogar ein detaillierter Nachvollzug der über Karten und geographische Fachliteratur durchaus verfügbaren Topographie der umkämpften Stadt war dem Verfasser meist gleichgültig: Es dominieren sehr allgemeine Beschreibungen Jerusalems, die Mauern der Stadt machen sich kaum anders bemerkbar als durch ihre Rolle als Orte des Angriffs- und Abwehrkampfes, allenfalls noch als Untersatz für die Teichoskopie. Ähnlich generisch sind die Beschreibungen der heiligen Stätten, gar nicht zu reden von Hinweisen auf Sitten und Gebräuche der einheimischen Bevölkerung. Und wie selbstverständlich besteht die Fiktion einer weitgehend ungehinderten sprachlichen Verständigung: Wenn beispielsweise der Heide Argant vor Gottfried spricht, ist die Kommunikation zwischen beiden umstandslos garantiert, denn "die Franken hatten bereits die Sprache Syriens gelernt“ (II.488/489: e perché i Franchi 


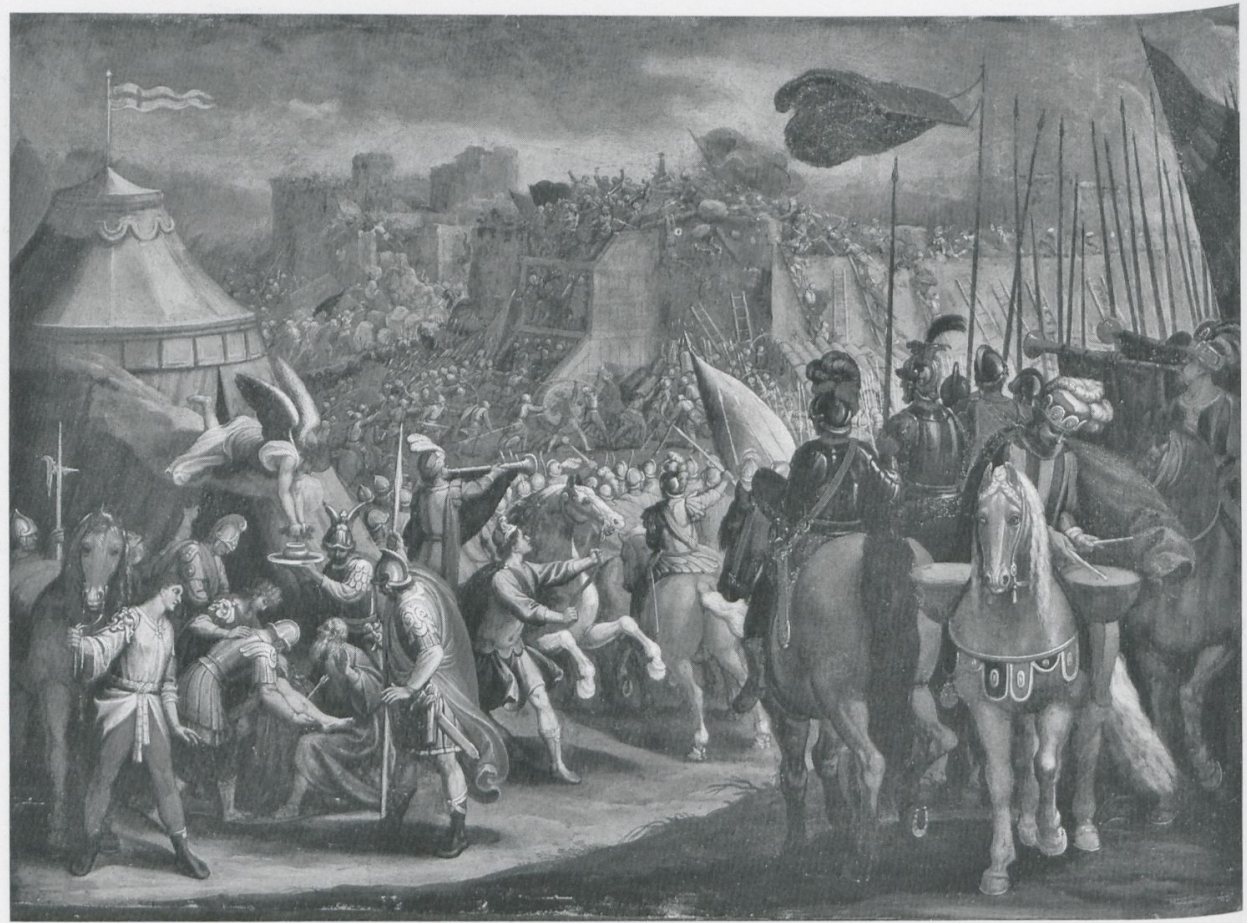

Abb. 7 Antonio Tempesta (und Werkstatt?), Der verwundete Gottfried wird von einem Engel geheilt, Florenz, Galerie Tornabuoni Arte, 1620er Jahre.

han già il sermon appreso/De la Soria, fu ciò ch'ei disse, inteso). Die entscheidende Differenz der von Tasso in sein Werk eingeführten Akteure wird durch den Gegensatz von Christen- und Heidentum konstitutiert, doch sogar auf dieser Ebene bleibt der ritterliche Ehrenkodex vielfach kulturelle Klammer zwischen Christen und „Pagani“, gar nicht zu reden davon, dass individuelle Verfehlungen oder emotionales Engagement auch einige der christlichen Protagonisten in ein moralisches Zwielicht stellen oder sie von der ,klaren` Fronstellung gegen die Heiden fortführen. ${ }^{29}$

Tempesta hat in seinen Gerusalemme-Serien die in der literarischen Vorlage eher verstreuten als gehäuften Anspielungen auf kulturelle Differenzen zwischen den Kreuzrittern und ihren militärischen Gegnern (d.h. Differenzen jenseits des vorausgesetzten elementaren religiösen Gegensatzes) aufgegriffen und gemäß seiner eigenen Erfahrung in der Darstellung ,verwandter' Sujets aus kirchlicher Propaganda, Geschichte und Topographie in Bilder übersetzt. Dies geschah vielfach stereotyp und - was angesichts der nicht spezifizierten Blicke auf die Baulichkeiten Jerusalems auffällt - sogar ohne Rückgriff auf seine eigene Arbeit an den Illustrationen der Terra Santa. Manchmal, z. B. in der Darstellung von Schilden und federgeschmückten Hüten oder Turbanen, scheint 
Abb. 8 Antonio Tempesta, Der verwundete Gottfried wird von einem Engel geheilt (Blatt aus der zweiten, Gerusalemme'-Folge),

Privatbesitz, 1620er Jahre.

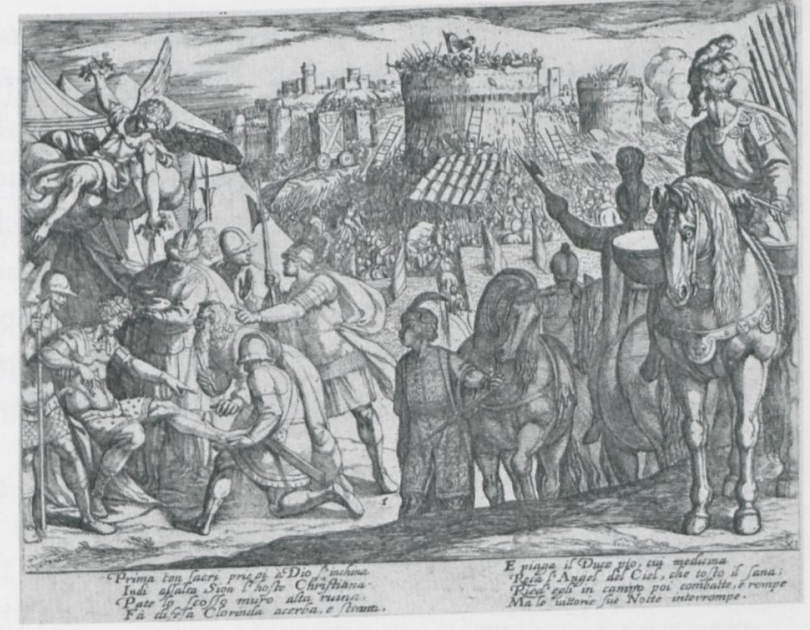

Tempesta vielleicht mit mehr Hintergrundwissen als etwa Castello operiert zu haben, obwohl er diese ihm als ,orientalisch ' geltenden Ausstattungsstücke wie selbstverständlich in die Zeit des Ersten Kreuzzugs zurückversetzte. Doch die übergreifende Funktion dieser Kostümierung ist deutlich: Während die Franken bei ihm eher antikisch-römisch und damit den damaligen Betrachtern ,vertraut ` daherkommen, hat er deren heidnischen Gegner solcherart exotisiert oder alterisiert.

Für Tempesta waren die beiden späten Tasso-Serien die Synthese eines ganzen Oeuvres, auch wenn einige dieser Kompositionen in Druckerschwärze ,ertrinken', weil die Ambition des Künstlers angesichts einer für solchen Detailreichtum noch nicht ganz entwickelten Radiertechnik wohl zu groß war. Die motivischen Anleihen bei antiken Monumenten und den Cinquecento-Kollegen von Vasari bis Castello - und auch der gemischte Modus - entsprechen durchaus dem eklektischen Ansatz von Tassos dichterischer Arbeit. Angesichts der vielfach ungeklärten Chronologie des malerischen Werks von Tempesta muss aktuell die Frage offen bleiben, ob einige dem Künstler zugeschriebene Gemälde mit Sujets aus der Gerusalemme den radierten Zyklen vorausgingen oder ihnen folgten, zum Beispiel eine Darstellung des verwundeten Gottfried in seinem Zelt, dem ein einschwebender Engel Medizin bringt, während im Hintergrund der Kampf um die Mauern von Jerusalem tobt ${ }^{30}$ (Abb. 7, Taf. XIV) - ein Bild, das kompositorisch den Radierungen zu Canto XI im zweiten ${ }^{31}$ (Abb. 8) und dritten ${ }^{32}$ Zyklus nahesteht. Sind Tempestas radierte Gerusalemme-Serien also mindestens teilweise graphische Wiederholungen von schon für Sammler gemalten Bildern oder dienten die Serien vielmehr gleichsam als Bestellkatalog für künftige Gemälde des Künstlers und seiner Werkstatt mit entsprechenden (wenn auch fast nie kompositorisch absolut identischen) Sujets? Wenn letzteres der Fall war, hat Tempesta selbst seiner Klientel in den Radierungen eine Art Baukasten zur Verfügung gestellt, aus dem sie sich ihren ganz persönlichen Gerusa- 
lemme-Wandschmuck zusammenstellen konnte. Die Tatsache, dass sich zahlreiche Detailzitate aus den Gerusalemme-Folgen Tempestas in der Malerei des 17. Jahrhunderts finden, dürfte daher schon durch diese vom Künstler selbst vorgesehene Funktion seiner Drucke bedingt sein. In den Gemäldegalerien der Epoche waren insbesondere Schlachten zwischen ,Christen' und ,Orientalen' beliebt, die nicht so leicht wie Darstellungen aus der Geschichte von Rinaldo und Armida auf eine bestimmte Textstelle Tassos bezogenen werden können, die aber doch als pittoreske Komplemente zu solchen narrativen Figurenbildern und womöglich auch zu ,exakten` Bildreportagen der noch lange anhalten kriegerischen Auseinandersetzungen mit den Osmanen gehängt worden sein dürften. 
1 Vgl. (mit älterer Literatur) E. Leuschner, Antonio Tempesta, Ein Bahnbrecher des römischen Barock und seine europäische Wirkung, Petersberg, Imhof, 2005, S. 504-508.

2 The Illustrated Bartsch (TIB), 35, New York, Abaris Books, 1984, S. 87: Titel der Vita des Antonius Abbas, Widmung an Cinzio Aldobrandini. TIB 37, New York 1984, S. 173: Titel der Ersten Monatsfolge, Widmung an Pietro Aldobrandini. Il Goffredo overo Gerusalemme liberata del Sig. Torquato Tasso, Rom, Gio. Ange. Ruffinelli, 1607. Il Goffredo overo Gerusalemme liberata del Sig. Torquato Tasso, Rom, Gio. Ange. Ruffinelli, 1601. G. B. Marino, Epistolario, hg. von A. Borzelli, 2 Bde., Bari, Laterza, 1911-12, Bd. I, S. 311.

J. Unglaub, Poussin's purloined letter, in "The Burlington Magazine", CXLII, 2000, S. 35-39. 37, 1999, S. 138-155. E. Leuschner, Drawings by Antonio Tempesta for a 'Gerusalemme Liberata', in "Master Drawings",

Als Ausnahme und bislang ausführlichste Interpretation von Gerusalemme-Darstellungen im Kontext der zeitgenössischen Konflikte mit den Osmanen und der mediceischen Kreuzzugsbestrebungen darf der Katalog L'arme e gli amori von 2001 gelten: L'arme e gli amori. La poesia di Ariosto, Tasso e Guarini nell'arte fiorentina del Seicento, Ausst. Kat., Florenz, Palazzo Pitti, hg. v. E. Fumagalli, M. Rossi und R. Spinelli, Florenz, Olschki, 2001 (zu Tempesta S. 112-130).

9 T. Staudacher, 'Volendo far la favola affettuosa'. Affektpoetik und Heidendarstellung bei Torquato Tasso, Wiesbaden, Harrassowitz Verlag, 2013.

10 Noch nicht unbedingt zu einem Antagonismus von Christentum und Islam, denn das hätte die tatsächliche Existenz zweier Religionen vorausgesetzt, während es doch nach zeitgenössischer christlicher Auffassung eigentlich um den Gegensatz von einzig wahrer Religion und Aberglauben oder Häresie ging. Die Begriffe „Islam“ und „Muslim“ wurden von christlichen Autoren der Zeit - und so auch von Tasso - selten bis nie gebraucht; weitaus häufiger waren Etiketten wie "infideli“, „pagani“ oder „Turchi“, vgl. Staudacher, 'Volendo far la favola affettuosa', S. 220.

11 Vgl. J. Kappel, Die Türkennot des Kaisers. Zu einigen Aspekten der Darstellung des Türkenkrieges (1593-1606) in der Hofkunst Rudolfs II, in Im Lichte des Halbmonds. Das Abendland und der türkische Orient, Ausst. Kat., Dresden, Staatliche Kunstsammlungen; Bonn, Bundeskunsthalle, hg. von den Staatlichen Kunstsammlungen Dresden, Leipzig, Ed. Leipzig, 1995, S. 124-133; E. Leuschner, Donau-Topographie und-Allegorie in der Türkenkriegspropaganda zwischen RudolfII. (1552-1612) und Leopold I. (1640-1705), in Barocke Kunst und Kultur im Donauraum, hg. v. K. Möseneder, M. Thimann und A. Hofstetter, 2 Bde., Petersberg, Imhof, 2014, Bd. I, S. 113 125.

12 Zu der laut Tasso durch den Anblick des Fremden hervorgerufenen „meraviglia“" vgl. Staudacher, 'Volendo far la favola affettuosa', S. 36.

13 Auch in Tempestas Gerusalemme werden - sicher ganz im Sinne Tassos - mehrfach göttliche Interventionen durch B-Handlungen im/aus dem Himmel dargestellt, z. B. der Gottfried heilende Engel in Canto XI.

14 E. Leuschner, Tempesta at the Capitoline, in "The Burlington Magazine", CXLI, 1999, S.618621.

15 G. Bosio, Istoria della Sacra Religione et Ill.ma Militia di S. Giovanni Gierosolimitano, Rom, Facciotti, 1602.

16 Nicht erfasst durch Adam von Bartsch, abgebildet in E. Leuschner, The Illustrated Bartsch 35 Commentary Part II, New York, Abaris Books, 2007, S. 219, Kat. Nr. 566.

17 Tempesta hat auch in seiner Darstellung des "Sieges von Goleta bei Tunis“ in der Serie der "Triumphe Kaiser Karls V.“, gestochen von Jacques de Gheyn (Hollstein Dutch \& Flemish 
Etchings, Engravings \& Woodcuts, vol. VII, S. 193, Nr. 4), die Romanitas des Kaisers dadurch herausgestrichen, dass er das Ereignis als quasi antike Belagerungs- und Schlachtenszene repräsentierte.

18 E. Leuschner und Ph. Rouillard, Metamorphoses of a Plate. The 'Battle of Las Navas de Tolosa' by Villamena after Tempesta, in "Print Quarterly", 19, 2002, S. 235-253.

19 E. Leuschner, 'Une Histoire telle que celle-ci, qui tient un peu du Roman'. Allegorie und Historie in Antonio Tempestas 'Infanten von Lara' und bei André Félibien, in "Marburger Jahrbuch für Kunstwissenschaft", 32, 2005, S. 203-243.

20 TIB 35, S. 61-85.

21 Z. B. Bernardino Poccetti im Palazzo Pitti, Sala di Bona (L'arme e gli amori, Ausst. Kat., S. 137) oder Pietro Tacca in der Darstellung der gefangenen Orientalen am Denkmal für Ferdinando de ${ }^{c}$ Medici in Livorno.

22 An dieser Stelle sei bereits auf den Forschungsbericht in der für 2019 vorgesehenen Publikation der Ergebnisse des DFG-Forschungsprojekts zur Typographia Medicea Orientale in ihrer Funktion als Organ des Kulturtransfers zwischen christlich-europäischen und muslimisch geprägten Kulturräumen in der Zeit um 1600 hingewiesen, die von Eckhard Leuschner und Gerhard Wolf herausgegeben werden wird.

23 Zu diesem Werk vgl. E. Leuschner und Y. Kouroshi, Topographie und Parteilichkeit. Die 'Terra Santa' des Bernardino Amico (Rom 1608, Florenz 1620) als Dokumentation der christlichen Stätten unter osmanischer Herrschaft, in "Revista de Historiografía", XI, 2, 2014, S. 67-86.

24 Diario Raimondis, Archivio di Stato di Firenze, Misc. Med. 272, fol. 7r.

25 S. J. Barnes, N. De Poorter, O. Millar und H. Vey, Van Dyck. A complete Catalogue of the Paintings, New Haven und London, Yale University Press, 2004, S. 203, Kat. Nr. II.62. Vgl. auch A. M. Piemontese, La Persia istoriata in Roma, Città del Vaticano, Biblioteca Apostolica Vaticana, 2014, S. 303 .

26 Orazioni Del Sig. Scipione Ammirato. A Diversi Principi. Intorno i preparamenti, che s'aurebbono a farsi contra la potenza del Turco, Florenz, Filippo Giunti, S. 159.

27 Zu Patrizi vgl. Leuschner, Allegorie und Historie, 2005, S.384, Anm. 102.

28 Auch wenn der Vergleich mit zeitgenössischen Jagddarstellungen unter Beteiligung von, Orientalen' anderer Künstler, vor allem Rubens, nahelegt, dass solche Bilder keineswegs ohne dezidierte Hinweise auf die zeitgenössischen Konflikten waren - vgl. U. Heinen, Antwerpen am Euphrat verteidigen. Rubens malt für Europa. Zur Vielfalt des frühneuzeitlichen Orientalismus, in Das Bild des Feindes. Konstruktion von Antagonismen und Kulturtransfer im Zeitalter der Türkenkriege, hg. v. E. Leuschner und T. Wünsch, Berlin, Mann, 2013, S. 355-448, hier S. 405-408.

29 Staudacher, 'Volendo far la favola affettuosa', S. 117 und passim.

30 Antonio Tempesta (und Werkstatt?), Der verwundete Gottfried wird von einem Engel geheilt, Öl auf Kupfer, $51 \times 71 \mathrm{~cm}$, Galerie Tornabuoni Arte, Florenz.

31 TIB 37, S. 102.

32 TIB 37, S. 122. 